\title{
Análisis de necesidades y planificación del curso en un grupo de estudiantes migrantes adultos de ELE
}

\author{
ANA GÓMEZ-PAVÓN DURÁN \\ Universitat de València \\ agopadu@uv.alumni.es
}

\begin{abstract}
Resumen: La escasez de materiales y métodos específicamente diseñados para la enseñanza de ELE a personas migrantes supone un obstáculo en la planificación del curso para los docentes que trabajan con este tipo de estudiantes cuyo principal objetivo en su aprendizaje del español es que esto les facilite la búsqueda de empleo y la integración en la sociedad de acogida. En este estudio se propone el análisis de necesidades como primer paso para decidir los contenidos de un curso de ELE para personas migrantes, y se ofrece la descripción de un cuestionario que se llevó a cabo como método de análisis de las necesidades de un grupo de alumnos de la fundación Cepaim en Valencia. En la descripción de los resultados de dicho análisis se explica también cómo contribuyó la información obtenida a partir del análisis a la planificación del curso.
\end{abstract}

Palabras clave: ELE, inmigrantes adultos, análisis de necesidades, planificación del curso.

\section{Needs Analysis and Course Design in a Group of Migrant Adult Students of SFL}

Abstract: The lack of specifically designed resources and methods for the teaching of Spanish as a Foreign Language (SFL) for migrants makes the task of course design more complex for teachers working with this type of students whose main objective in their learning of the Spanish language is to become integrated into the Spanish society and find employment. This study proposes the needs analysis as the first step for the course design of a Spanish course for migrants. It offers the description of a questionnaire that was carried out as a needs analysis method in a group of students from the Cepaim Foundation in Valencia. The description of the results of the aforementioned analysis includes an explanation of how the information obtained by means of the analysis contributed to the course design.

Keywords: Spanish as a foreign language (SFL), SFL for adult migrants, needs analysis, course design.

\section{El concepto de análisis de necesidades y su relevancia en la enseñanza de ELE para personas migrantes}

La enseñanza de ELE para personas migrantes adultas supone un gran reto para el docente que se enfrenta a esta situación. La gran incógnita para quienes trabajan con este tipo de alumnado es qué enseñar y cómo enseñarlo, y la escasez de materiales diseñados específicamente para este colectivo no resulta de gran ayuda. Por esta razón, en este artículo se propone el análisis de necesidades como primer paso para decidir cuáles serán los contenidos que se impartirán durante el curso y de qué manera se deberán enseñar. Para ilustrar mejor esta propuesta, en los apartados que siguen a esta primera introducción 
se describe una experiencia práctica que consistió en un análisis de necesidades realizado a un grupo de 14 estudiantes migrantes adultos que asistían al curso de español ofrecido por la fundación Cepaim ${ }^{1}$ en Valencia.

El concepto de análisis de necesidades puede llevar al lector a plantearse en primer lugar qué se debe entender exactamente por necesidades. Castellanos, retomando la idea de análisis de necesidades planteada y desarrollada inicialmente por Munby (1978) y West (1994), define las necesidades como «las demandas o exigencias propias de una situación de comunicación, es decir, todo aquello que la situación requiere para que el hablante pueda actuar de un modo efectivo en esa situación» (2010: 3). No obstante, como explica este autor, las necesidades de los estudiantes son siempre cambiantes y es un riesgo concebirlas como algo estático y común a todas las personas. Por esta razón, afirma que el análisis de necesidades es algo que debe llevarse a cabo no solo al comienzo de las clases para planificar los contenidos, sino a lo largo de todo el curso para poder readaptar estos y conseguir que se ajusten lo máximo posible a las demandas de las diferentes situaciones que se encuentran en el aula (2010:3).

En el aula de ELE para personas migrantes es también conveniente el análisis de necesidades como primer paso para el diseño del programa del curso o la revisión y readaptación de contenidos (García 2008: 46; Cividanes y Arza 2011: 195; Toledo 2016: 83). En una encuesta destinada a conocer si los profesores de la fundación Cepaim se servían de este tipo de análisis, el $89 \%$ de un total de 9 participantes afirmó que sí lo hacía, por medio de métodos como la observación en el aula, la elaboración de cuestionarios o las entrevistas con los estudiantes ${ }^{2}$.

Este interés por conocer las necesidades de los estudiantes migrantes a la hora de decidir los contenidos que se impartirán durante el curso se debe a que este tipo de alumnado presenta una serie de características y objetivos que difieren de los de los estudiantes estándar de ELE, como afirman Cividanes y Arza:

A una clase de ELE para inmigrantes asistirán alumnos de diferentes nacionalidades, culturas, lenguas y niveles educativos. Por este motivo, necesitaremos un tratamiento educativo diferente al habitual, ya que precisan aprender español de una forma rápida y funcional. Consecuentemente, es indispensable identificar las necesidades e intereses de los alumnos, definir el objetivo que se pretende conseguir y diseñar un programa que facilite la consecución de estos propósitos (2011: 195).

Asimismo, es muy frecuente que en las aulas de ELE para inmigrantes se incorporen al grupo alumnos nuevos durante el curso y que otros deban abandonar las clases, lo que significa que el carácter cambiante de las necesidades del grupo que menciona Castellanos (2010: 3) es mucho más pronunciado en este tipo de estudiantes.

Así pues, debido a estas diferencias de la enseñanza de ELE para migrantes con respecto a la enseñanza de ELE estándar, los manuales diseñados para el aula común de ELE no siempre serán adecuados para el alumnado migrante, por lo que el profesor deberá readaptar los materiales de los que dispone o elaborar recursos nuevos que sí respondan

\footnotetext{
${ }^{1}$ Para conocer más sobre la fundación Cepaim y sus programas ver http://cepaim.org/

2 Por motivos de espacio, no se han incluido en este artículo los detalles de los resultados de dicha encuesta. Para solicitar los resultados de la encuesta, contactar con DIRECCIÓN DE CORREO ELECTRÓNICO.
} 
a las necesidades de sus estudiantes, tarea que se vería facilitada por la realización de un análisis de necesidades que sirva como punto de partida.

\section{Métodos de análisis empleados para conocer las necesidades de los estudiantes de la Fundación Cepaim}

A fin de conocer las necesidades de un grupo de estudiantes migrantes adultos en la fundación Cepaim (Valencia) y poder planificar los contenidos del curso se llevó a cabo un análisis de necesidades por medio de tres métodos diferentes: un cuestionario, la observación en clase y el diálogo entre los estudiantes y la profesora.

Los participantes de los cursos de español en la fundación Cepaim se distribuyen en diferentes grupos, en función del nivel, de la disponibilidad de las aulas y del número de profesores que se ofrecen voluntariamente para impartir clase. Normalmente los estudiantes se distribuyen entre un grupo de alfabetización y otro grupo mayor que se subdivide en niveles iniciales, intermedios y avanzados. El grupo estudiado pertenecía a un nivel intermedio, que se correspondería con un A2-B1, aproximadamente y estaba integrado en el momento del estudio por un total de 14 alumnos, los cuales se sometieron al análisis en distintos momentos del curso (entre los meses de octubre y diciembre de 2018). Este grupo se componía por 3 mujeres y 11 hombres, que se encontraban entre los 18 y los 50 años de edad, aproximadamente.

Es importante destacar que, a lo largo del curso, los integrantes del grupo iban cambiando. Algunas personas tuvieron que abandonar el curso mientras que otras se fueron incorporando en distintos momentos, de forma que los métodos de análisis antes mencionados se aplicaron no solo el primer día de clase, sino también cada vez que un nuevo alumno empezaba a formar parte del grupo. De esta forma los contenidos podían modificarse si era necesario y adaptarse lo máximo posible a las necesidades de cada uno de los estudiantes.

\subsection{Cuestionario}

El cuestionario (ver Anexo) consiste en diez preguntas de diversa índole. Debido a las diferencias de nivel en el aula, algunos estudiantes encontraron dificultades para comprender las preguntas, aunque generalmente no presentaron grandes problemas. Para facilitarles la compresión, se leyeron y comentaron las preguntas y se resolvieron las dudas correspondientes antes de comenzar el cuestionario.

Las cuatro primeras preguntas son de carácter personal (nombre, país de origen, lengua materna y otras lenguas que hablas) y permiten al docente conocer mejor al estudiante y el contexto cultural del que proviene. Además de esto, la respuesta a la pregunta Otras lenguas que hablas puede indicar si el estudiante se ha enfrentado con anterioridad al aprendizaje de una lengua extranjera. Asimismo, si el estudiante conoce alguna lengua europea o románica (como el francés en el caso de algunos estudiantes de origen africano) y que, por consiguiente, guarde una mayor similitud con el español, puede significar que encontrará más facilidades que algunos de sus compañeros en ciertos momentos de su aprendizaje.

Las preguntas 5 y 6 se realizan con intención de obtener una idea aproximada del nivel de lengua del estudiante. Generalmente, un estudiante que lleve más tiempo en España o que haya cursado antes un curso de lengua española tendrá un conocimiento mayor de la lengua. No obstante, esta información se ha de complementar con la que se obtiene a partir de la observación por parte del docente, que permite conocer con más detalle cuáles 
son las carencias o dificultades concretas que presenta el estudiante y que deberán ser resueltas a lo largo del curso. Además, en ocasiones podemos encontrar estudiantes que, a pesar de haber permanecido varios años en España, no presentan el nivel que se esperaría de ellos puesto que su contacto con hablantes nativos es bastante limitado. Del mismo modo, también puede haber estudiantes cuyo nivel de integración en la sociedad de acogida es muy elevado y que presentan un gran dominio de la lengua a pesar de haber permanecido menos tiempo en España. Este tipo de datos, que no se observarían en el cuestionario, se pueden obtener a partir de la observación, el diálogo con los estudiantes o una prueba de nivel.

La pregunta número 7 va destinada a conocer aspectos como la percepción que tienen los estudiantes de su propia interacción con los hablantes nativos, las dificultades o inseguridades que esta situación les genera, o cuestiones de carácter pragmático relacionadas con la cortesía, las costumbres, las formas de dirigirse al interlocutor, etc. También se ofrece la posibilidad de que el estudiante añada otros problemas que encuentra a la hora de comunicarse en español.

Dado que las necesidades se relacionan con las situaciones comunicativas (Castellanos 2010: 3), en la pregunta 8 se pretende conocer las situaciones comunicativas en las que los estudiantes consideran que deben saber desenvolverse o en las que encuentran más dificultades, planteadas desde los contextos a los que se enfrentan en su día a día, como la búsqueda de empleo o vivienda, el uso de transporte público o la cumplimentación de documentos. Al igual que en la pregunta anterior, el estudiante puede añadir otras situaciones en las que encuentra problemas de comunicación.

La pregunta 9 permite conocer las necesidades subjetivas de los estudiantes; esto es, sus deseos de aprendizaje. Para que sirvan de guía al estudiante, se sugieren algunas posibles respuestas al estudiante: gramática, vocabulario, costumbres españolas o valencianas, política/historia/cultura de España.

Finalmente, en la pregunta 10 se pide al estudiante que elabore una pequeña producción escrita de temática sencilla que permitirá al docente, a través de la observación de las distintas respuestas, conocer de forma aproximada algunas carencias o dificultades del grupo que guarden relación con la competencia escrita.

\subsection{La observación en clase y el diálogo profesor-alumno}

Además del cuestionario, la profesora decidió aplicar otros métodos de análisis que permitieron complementar la información obtenida a partir de las preguntas que se han comentado anteriormente. El primero de ellos fue la observación en clase, pues, de acuerdo con Castellanos, «constituye una técnica ideal para constatar las necesidades del grupo y de cada uno de los alumnos» y permite al profesor examinar «el comportamiento de los alumnos con respecto a la adquisición de la competencia comunicativa, los instrumentos lingüísticos que necesitan, y los medios y métodos que mejor favorecen su estilo de aprendizaje» (2010: 30). En el caso descrito, la observación en clase se realizó principalmente a partir de las intervenciones orales de los estudiantes, las interacciones orales entre ellos y las producciones escritas que elaboraron no solo como parte del cuestionario, sino también en otros momentos a lo largo del curso.

Asimismo, se utilizó también el diálogo profesor-alumno en los primeros días de clase y durante el desarrollo del curso la profesora como método para conocer nuevas necesidades del grupo, pues es un medio que «sirve de estímulo para los alumnos»y 
permite al profesor conocer aquellos «temas, actividades, destrezas, estilos de aprendizaje, etc., que los alumnos están interesados en desarrollar» (Castellanos 2010: 30). Gracias al diálogo en clase se pudieron comentar también las respuestas que los estudiantes habían ofrecido en el cuestionario.

\section{Resultados del análisis y su aplicación a la planificación de los contenidos del curso}

A continuación, se muestran los resultados obtenidos a partir de los análisis realizados a los integrantes del grupo estudiado. Asimismo, se ofrece un comentario de las respuestas a las preguntas del cuestionario ${ }^{3}$, indicando de qué manera estas pueden repercutir en la planificación de los contenidos del curso y cómo se complementó la información obtenida a partir de dichas respuestas con la observación en el aula por parte de la profesora y del diálogo entre esta y su grupo de estudiantes.

Las respuestas a la pregunta número 2, País de origen, indican que los 14 integrantes del grupo procedían de 11 países diferentes: Armenia, Ucrania, Costa de Marfil, Ghana, Guinea Conakry, Marruecos, Mauritania, Nigeria, Senegal, India y Pakistán. Esto significa que, en este caso, al igual que suele suceder en la mayoría de las aulas de ELE para personas migrantes, encontramos una gran diversidad cultural en el aula que el docente puede aprovechar para trabajar la competencia intercultural. Sobre todo para las primeras clases se planificaron actividades destinadas a trabajar dicha competencia, que contribuyeron a crear en el aula una mayor conciencia sobre los distintos contextos culturales y un mejor ambiente de trabajo. Asimismo, el desarrollo de la competencia intercultural de los alumnos a lo largo de todo el curso puede contribuir también a facilitarles el proceso de integración en la sociedad de acogida, pues no debemos olvidar que esta es una de las prioridades de los estudiantes migrantes de ELE.

De modo similar, las respuestas a las preguntas 3. Lengua materna y 4. Otras lenguas que hablas, reflejan la gran diversidad lingüística del aula, que también puede ser tratada en actividades destinadas a trabajar la competencia intercultural. En el grupo estudiado encontramos hasta 10 lenguas maternas distintas: wolof, soninké, ucraniano, hindi, urdu, bereber, árabe, francés, armenio e inglés. Además, a excepción de un alumno, los 13 restantes podían hablar otras lenguas diferentes del castellano y su lengua materna, y el $50 \%$ de ellos tenía el inglés o el francés como segunda lengua.

En cuanto a la pregunta 5. ¿Cuánto tiempo llevas en Valencia?, los resultados muestran que el 64\% de los estudiantes llevaba en España un año o menos, mientras que el $36 \%$ llevaba más de un año en el país. En la pregunta 6. ¿Has ido a clase de español antes?, un $36 \%$ de los alumnos respondieron que habían asistido a un curso de español con anterioridad, mientras que el $64 \%$ no lo había hecho nunca. Esto puede indicar que, como sucede con frecuencia en las aulas de ELE para personas migrantes adultas, dentro un mismo grupo puede haber diferencias de nivel. Por tanto, a la hora de planificar los contenidos del curso, será necesario identificar cuáles son estas diferencias por medio de métodos más adecuados, como una prueba de nivel, y tratar de satisfacer las necesidades de todos los miembros del grupo al mismo tiempo. Más concretamente, en el caso estudiado, gracias a la observación de la profesora de sus producciones escritas, sus

\footnotetext{
${ }^{3}$ Se muestran los resultados de la pregunta 2 a la 9. En la pregunta número 1 se pedía a los estudiantes que indicaran su nombre y, por motivos de confidencialidad y por la escasa relevancia de esta información para este artículo, no se han facilitado las respuestas a esta pregunta. La pregunta 10 consistía en una pequeña producción escrita, y por motivos de espacio tampoco se comparten en este artículo las distintas respuestas.
} 
intervenciones orales y sus fallos o errores en los ejercicios propuestos, fue posible concretar con más precisión cuáles eran las carencias de cada estudiante.

A pesar de las diferencias de nivel dentro del grupo, fue posible realizar actividades e impartir contenidos que fueran beneficiosos para todos los estudiantes. Por ejemplo, a partir de la observación se detectó que, en términos de gramática, los estudiantes que llevaban más tiempo en España presentaban problemas o errores fosilizados en aspectos básicos como la concordancia de género y número, las conjugaciones de verbos en tiempos sencillos o los usos de los pronombres. También cometían muchos errores de ortografía o, en relación con algunas áreas temáticas, presentaban un léxico muy reducido. Por lo tanto, para ayudar a los estudiantes de más nivel con dichas dificultades, se explicaban desde el principio estos conceptos, de forma que ellos podían así resolver sus dificultades mientras que sus compañeros de nivel inferior adquirían esos conocimientos por primera vez.

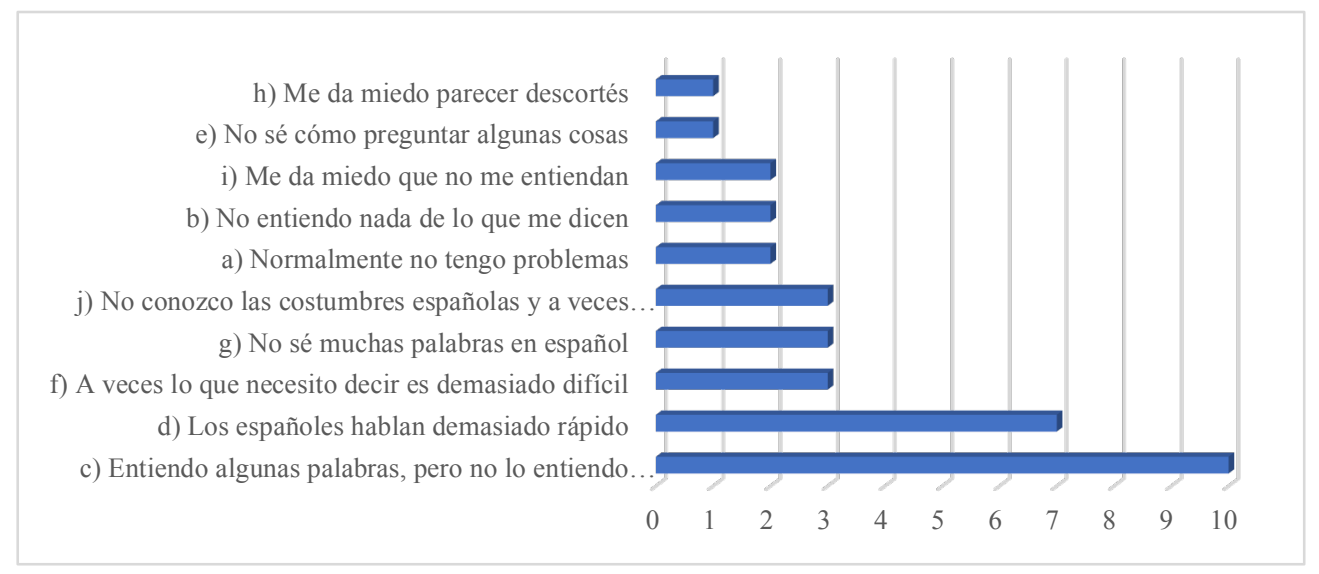

Gráfico 1. Frecuencia de cada opción de respuesta para la pregunta número 7 del cuestionario.

Como muestra el gráfico anterior, en la pregunta 7. Cuando intentas hablar en español con otras personas, ¿qué problemas tienes normalmente?, las respuestas que se marcaron con más frecuencia fueron la c) y la d). Ambas respuestas están relacionadas con la comprensión oral y, al parecer, es la competencia que los estudiantes consideran más importante para que la interacción oral con los hablantes nativos sea exitosa, o al menos, la que perciben como más dificultosa o problemática a la hora de comunicarse en español. En las respuestas que añadieron los estudiantes y que no estaban entre las opciones, aparecen también otras destrezas comunicativas, como la expresión escrita y oral o la comprensión lectora.

Por tanto, en la planificación del curso fue necesario dedicar una mayor atención a estos aspectos, y especialmente a las destrezas orales, no solo para conseguir desarrollar y mejorar esta competencia en los estudiantes, sino también para reducir la ansiedad o la frustración que pudiera provocarles esta dificultad en la interacción oral en español. La integración en la sociedad de acogida es una de las prioridades de este tipo de alumnado, y por tanto es conveniente prestar especial atención a todo aquello que pueda dificultar a los estudiantes migrantes lograr este objetivo.

Es importante tener en cuenta que la respuesta c), además de producirse por la falta de comprensión oral, también puede ser causada por poseer un léxico reducido. Esto sucede 
también con las opciones f) y g) que, junto con la opción j), fueron las que se marcaron con más frecuencia después de las opciones c) y d). Por lo tanto, a la hora de planificar los contenidos del curso, el léxico fue un aspecto al que también se le dio una gran importancia, como se habría hecho en cualquier otro curso de ELE. No obstante, es importante plantearse qué tipo de léxico debe enseñarse en el aula de ELE para migrantes y tratar de que se ajuste lo máximo posible a las necesidades de los estudiantes. Más concretamente, para este grupo se pensó que lo más acertado era incluir en los contenidos del curso un léxico relacionado con las situaciones comunicativas a las que se iban a enfrentar los estudiantes en su día a día. Para conocer cuáles eran estas situaciones se realizó la pregunta 8 , que se comenta a continuación.

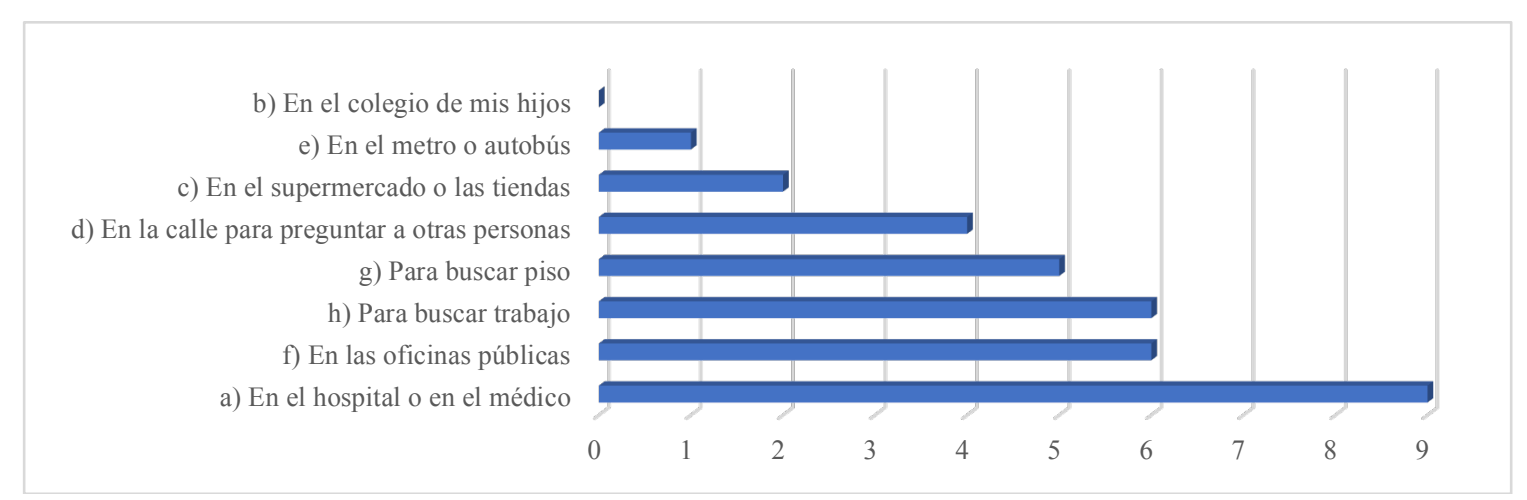

Gráfico 2. Frecuencia de cada opción de respuesta para la pregunta número 8 del cuestionario.

La pregunta 8. ¿En qué momentos o lugares necesitas ayuda para comunicarte en español? permite conocer las situaciones comunicativas en las que los estudiantes consideran que deben saber desenvolverse o en las que encuentran más dificultades. Como se observa en el gráfico 2, la respuesta marcada con más frecuencia fue la a), seguida de la f), la h) y la g). Por tanto, en el momento de escoger los contenidos que se debían impartir en este curso, se decidió que una parte del léxico debía estar relacionada con los contextos de salud y centros de salud, cumplimentación de documentación, búsqueda de empleo y búsqueda de vivienda, principalmente. Esto no significa que no se haya facilitado otro tipo de vocabulario a los estudiantes durante el curso, pero es cierto que, debido a la situación en la que se encuentran las personas migrantes y las urgencias que esta les genera, es importante intentar que los contenidos que se les imparte sean lo más prácticos posible para su situación diaria.

Además de para concretar el tipo de léxico que se debe impartir durante el curso, conocer las situaciones comunicativas más importantes para los estudiantes puede ayudar también a decidir qué funciones comunicativas son más prioritarias para ellos. Por tanto, se les dio más importancia a aquellas funciones comunicativas que fueran a necesitar en estas situaciones, mientras que las demás quedaron en un segundo plano. 


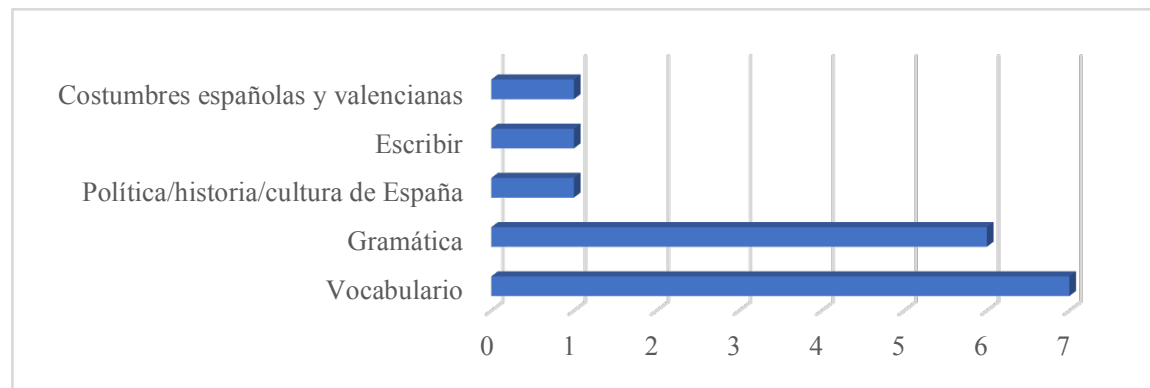

Gráfico 3. Frecuencia de las distintas respuestas ofrecidas por los estudiantes en la pregunta 9.

Finalmente, en la pregunta 9. ¿Qué te gustaría aprender en las clases de español? Por ejemplo: gramática, vocabulario, costumbres españolas o valencianas, política/historia/cultura de España..., los estudiantes pudieron expresar sus deseos de aprendizaje. La respuesta más repetida fue vocabulario, seguida de gramática.

Al parecer, estos son los aspectos que los estudiantes más deseaban aprender. Aunque es importante conocer las necesidades subjetivas de los estudiantes y tratar de satisfacerlas en la medida de lo posible, es tarea del docente decidir también cuáles son los contenidos más relevantes para los estudiantes y cómo deben impartirse. Por este motivo, la profesora decidió incluir también en el programa del curso actividades destinadas a desarrollar la competencia cultural por ser una destreza de gran utilidad en el proceso de integración de las personas migrantes, a pesar de que los estudiantes no lo consideraron un factor tan relevante, quizás porque desconocían su importancia al no tratarse de un aspecto al que se le preste demasiada atención en los modelos educativos tradicionales.

\section{Conclusiones}

El análisis de necesidades aplicado a este grupo de estudiantes y la información obtenida a partir de las respuestas de los alumnos al cuestionario facilitaron enormemente la decisión de la profesora sobre los contenidos que se debían incluir en el programa y el diseño de materiales para el curso. Asimismo, la observación y el diálogo profesoralumno complementaron esta información y permitieron un conocimiento más detallado de las necesidades de dicho grupo. Por tanto, de este artículo se espera que pueda servir de guía para aquellos docentes de español para personas migrantes que necesiten una primera aproximación de las necesidades de sus estudiantes antes de comenzar a planificar el programa del curso.

También cabe decir que, aunque el cuestionario descrito resultó de gran utilidad en este caso en particular, este podría admitir alguna mejora e incluir preguntas que permitieran obtener una información aún más precisa sobre las necesidades de los estudiantes. Por ejemplo, en futuras ocasiones sería interesante conocer, además de las situaciones comunicativas a las que el alumno considera que deberá enfrentarse, las funciones comunicativas que el estudiante considera prioritarias, como vemos en Toledo (2016). Asimismo, la inclusión de preguntas que incorporaran aspectos gramaticales o léxicos permitiría un conocimiento más preciso de las carencias del estudiante en este sentido, pues la pregunta en la que se pide al alumno que elabore una pequeña producción escrita puede resultar insuficiente para este propósito. 
Finalmente, es necesario reflexionar sobre las dificultades que presentan el aprendizaje y la enseñanza de ELE para inmigrantes y si los métodos de los que se disponen son suficientes para superarlas. Es cierto que contamos con un gran número de materiales y trabajos de investigación orientados a la enseñanza de ELE para inmigrantes que sin duda resultan de gran utilidad para quienes trabajan con este tipo de estudiantes, como los que se recopilan en los trabajos de Villalba y Hernández (2008) o Cabrera (2012). Sin embargo, las diferencias de nivel en los grupos, la irregularidad con la que asisten los estudiantes a las clases y la apremiante necesidad de integración social de los alumnos, entre otras circunstancias, exigen una gran flexibilidad y adaptabilidad de los materiales, así como un profundo conocimiento de las necesidades del grupo por parte del docente, destrezas que no siempre se pueden alcanzar fácilmente. Por consiguiente, la autora considera que debe continuar la actividad investigadora en este campo, especialmente la destinada a detectar las principales necesidades de los alumnos migrantes de ELE, ya que podría resultar en la creación de nuevos métodos y materiales que sin duda no solo facilitarían enormemente el trabajo a los profesores de español para personas migrantes, sino que también contribuirían a un mejor aprendizaje del español y, en consecuencia, una mayor integración por parte de las personas inmigrantes en España.

\section{Bibliografía}

CAstellanos Vega, Isidoro (2010). «Análisis de necesidades y establecimiento de objetivos», Monográficos MarcoELE, 10, 23-35. Recuperado el 28/4/2019, de: https://marcoele.com/descargas/expolingua_2002.castellanos.pdf.

CABRERA MONTESDEOCA, Carmen Delia (2012). La alfabetización de inmigrantes adultos en ELE. Una experiencia en las clases de español para ágrafos. Trabajo final de Máster. Las Palmas: Universidad de las Palmas de Gran Canaria. Recuperado el 14/09/2019 de: http://www.educacionyfp.gob.es/dam/jcr:8a4c0f7f-2703-4fea-9f9ee53de3c6c2c9/2013-bv-14-02carmen-delia-cabrera-pdf.pdf.

CividAnes Pereira, Romina y Montserrat ARZA Rodríguez (2011). «¿Por qué tiza y fotocopia? La creación de actividades interactivas para la enseñanza de ELE a inmigrantes». En C. Hernández, A. Carrasco, E. Álvarez (eds.); La Red y sus aplicaciones en la enseñanza-aprendizaje del español como lengua extranjera (pp. 195-204). Valladolid: Asociación para la Enseñanza del Español como Lengua Extranjera. Recuperado el 28/4/2019, de: https://cvc.cervantes.es/ensenanza/biblioteca_ele/ asele/asele_xxii.htm.

GARCÍA MATEOS, Crescencia (2008). «La enseñanza de la L2 para inmigrantes adultos: necesidades, estrategias, dificultades y maneras de aprender». En A. Ríos Rojas y G. Ruiz Fajardo (ed.); Didáctica del Español como $2^{a}$ Lengua para Inmigrantes (pp. 38-56). Sevilla: Universidad Internacional de Andalucía. Recuperado el 14/09/2019 de: https://dspace.unia.es/bitstream/handle/10334/128/2008_didactica.pdf?sequence=4\&isA llowed $=\mathrm{y}$.

MunBy, John (1978). Communicative Syllabus Design. Cambridge: Cambridge University Press.

Toledo VEGA, Gloria (2016). «Propuesta didáctica para la enseñanza de español como segunda lengua a inmigrantes haitianos en Chile», Lengua y migración, 8:1, 81-103. Recuperado el 14/09/2019 de: http://lym.linguas.net/Download.axd?type=Article Item\&id=162. 
VILlALBA, Félix y Maite HERNÁNDEZ (2008). «Referencias bibliográficas sobre L2 e inmigración en España (I)», Recursos para la investigación en adquisición/aprendizaje de segundas lenguas, 2, 10-69. Recuperado el 14/09/2019 de: http://segundaslenguaseinmigracion.com/Investigaciones.html.

WEST, Richard (1994). «Needs Analysis in Language Teaching», The International Abstracting Journal for Language Teachers and Applied Linguistics, 27: 1.

\section{Anexo}

1. Nombre:

2. País de origen:

3. Lengua materna:

4. Otras lenguas que hablas:

5. ¿Cuánto tiempo llevas en Valencia?

6. ¿Has ido a clase de español antes? Si has respondido sí, ¿dónde has recibido clases de español y durante cuánto tiempo?

7. Cuando intentas hablar en español con otras personas, ¿qué problemas tienes normalmente? Puedes elegir más de una respuesta.

a) Normalmente no tengo problemas.

b) No entiendo nada de lo que me dicen.

c) Entiendo algunas palabras, pero no lo entiendo todo.

d) Los españoles hablan demasiado rápido.

e) No sé cómo preguntar algunas cosas.

f) A veces lo que necesito decir es demasiado difícil.

g) No sé muchas palabras en español.

h) Me da miedo parecer descortés o no respetar a otras personas.

i) Me da miedo que no me entiendan.

j) No conozco las costumbres españolas y a veces no sé cómo actuar en algunas situaciones.

Si quieres puedes escribir otros problemas:

8. ¿En qué momentos o lugares necesitas ayuda para comunicarte en español? Puedes elegir más de una respuesta.

a) En el hospital o el médico.

b) En el colegio de mis hijos.

c) En el supermercado o las tiendas.

d) En la calle para preguntar a otras personas.

e) En el metro o autobús.

f) En las oficinas públicas (policía, extranjería...)

g) Para buscar piso.

h) Para buscar trabajo.

Si quieres puedes escribir otros lugares o situaciones en las que necesitas ayuda:

9. ¿Qué te gustaría aprender en las clases de español? Por ejemplo: gramática, vocabulario, costumbres españolas o valencianas, política/historia/cultura de España...

10. Escribe a continuación qué es lo que más te gusta de Valencia y lo que menos te gusta. Explica por qué: 